\title{
Diáspora salvadoreña: identidades y mapas culturales en el ciberespacio
}

José Luis Benítez

Universidad Centroamericana ‘José Simeón Cañas’ (UCA), San Salvador. Correo electrónico: joselu70@gmail.com

\section{Recibido: abril de 2008 / Aceptado: mayo de 2008}

EL FENÓMENO DE LA MIGRACIÓN INTERNACIONAL PLANTEA NUEVOS desafíos de investigación al campo académico de la comunicación. En esta perspectiva, algunos estudios de comunicación han analizado el papel de los medios de comunicación y sus narrativas mediáticas sobre la migración, la construcción de imaginarios sociales acerca de la experiencia de emigrar, y la importancia de las nuevas tecnologías en las prácticas cotidianas de los inmigrantes. En este contexto, este artículo explora algunos contenidos y formatos que grupos y comunidades migrantes salvadoreñas construyen desde sitios y páginas web, usos de medios de comunicación local y nacional accesibles desde el Internet, y nuevas plataformas de redes sociales como los blogs, Myspace, Facebook, YouTube, entre otros. Por tanto, este artículo intenta dibujar algunos trazos de mapas culturales y expresiones de identidades colectivas que la diáspora salvadoreña construye día a día en el ciberespacio.

Palabras clave: diáspora salvadoreña / comunicación transnacional / Internet y migrantes / medios de comunicación

\section{Introducción}

Los procesos de migración internacional han generado nuevas dinámicas económicas, sociales, políticas y culturales en las sociedades tanto emisoras como receptoras de flujos masivos de personas. De hecho, la migración internacional es considerada como una dimensión constitutiva de la globalización (Koser, 2007:28). Al menos hay tres aspectos en los cuales se interrelaciona la globalización con las dinámicas de migración internacional. Primero, la reconfiguración de nuevos mercados laborales y las demandas de mano de obra en países desarrollados. Segundo, la visibilidad que adquieren en el plano global las desigualdades socio-económicas entre países y regiones en el mundo, particularmente en el nivel de ingresos y de calidad de vida de las personas. Tercero, estas condiciones replantean la búsqueda de opciones sustentables y coherentes al tema de la pobreza y la necesidad de nuevos modelos y formas de desarrollo humano.

Los procesos contemporáneos de migración internacional también generan nuevas dinámicas de vinculación y prácticas transnacionales; es decir, acciones sociales que se desarrollan entre al menos dos Estados nacionales y que se apoyan, particularmente, en las 
nuevas tecnologías de información y comunicación. De esta forma se habla hoy en día de la construcción de un campo social o espacio social transnacional por parte de los migrantes y sus comunidades de origen (Pries, 2001:18). Así, se puede identificar una serie de procesos y prácticas económicas, políticas, sociales, culturales, religiosas y comunicacionales que establecen, nutren y transforman las redes de comunidades migrantes. Además, esta noción de procesos transnacionales pone en cuestión ideas tradicionales de absorción, integración o asimilación cultural que se han tendido a estudiar en los procesos de migración internacional. Este campo transnacional posibilita que muchos inmigrantes, ya sean documentados o no, puedan mantener y reproducir vínculos familiares, sociales y simbólicos con sus comunidades y países de origen.

Desde el campo académico de la comunicación, el fenómeno de la migración internacional ha sido objeto de diferentes investigaciones que intentan comprender las vinculaciones de la comunicación interpersonal y colectiva con la experiencia de la migración. Al menos hay tres áreas en las cuales se pueden delinear los aportes que la comunicación ha hecho a los estudios sobre migración internacional, tanto en América Latina como en otras regiones del mundo. Un área de interés ha sido estudiar el papel de los medios de comunicación en la construcción y circulación de narrativas, discursos y visiones acerca de la migración. Otro ámbito de investigación ha buscado analizar la formación de representaciones mediáticas y reproducción de imaginarios simbólicos que se fabrican en torno a la migración y sus personajes: los coyotes, los policías, los migrantes, los oficiales de migración, los empleadores, etc., y cómo se cuentan y se representan las experiencias de migración. Una tercera área de investigación ha sido explorar los usos y las significaciones socioculturales que las nuevas tecnologías de información y comunicación tienen en la vida cotidiana de los migrantes y de sus comunidades de origen, particularmente el uso del Internet, teléfonos celulares, videoconferencias y videojuegos. En este último punto, también se ha prestado atención al fenómeno de la brecha digital y de qué maneras estas desigualdades en el acceso y usos de las nuevas tecnologías se manifiestan en el nivel local, nacional, transnacional y global.

Desde esta perspectiva, este artículo examina las representaciones, narrativas y expresiones culturales que los inmigrantes salvadoreños construyen en el ciberespacio. El Internet no puede dejar de analizarse como un medio de comunicación -para algunos con mayores posibilidades de democratización y amplia participación- desde el cual se construyen representaciones socioculturales, historias populares y sentidos sociales de las experiencias de migración. Se plantean algunas reflexiones acerca de las relaciones entre estudios de la diáspora y el campo de la comunicación. Finalmente, se perfilan algunos elementos de lo que se podría denominar mapas culturales en el ciberespacio, desde los cuales se puede profundizar y ampliar este tipo de análisis para otros grupos o comunidades de migrantes centroamericanos.

\section{Algunos datos sobre la diáspora salvadoreña}

De acuerdo al reporte de 2007 del Ministerio de Relaciones Exteriores de El Salvador, un total de 2.2 millones de salvadoreños viven fuera del país. Esto significa que de los 5.8 millones de salvadoreños, cerca del 35\% reside en el exterior. El número más importante de inmigrantes se encuentra en los Estados Unidos, en donde hay 1, 842,100 salvadoreños 
que se concentran primordialmente en el estado de California y el área metropolitana de Washington D.C. Otros países donde se encuentran números significativos de migrantes salvadoreños son: Canadá $(135,500)$, México $(36,049)$, diferentes países de Centroamérica y el Caribe $(137,449)$, Italia $(32,130)$, Australia $(18,755)$, España $(3,200)$ y Suecia $(2,320) .{ }^{1}$

Una pregunta importante que algunos autores se plantean es ihasta qué punto esta gran cantidad de salvadoreños que han emigrado hacia diferentes países puede ser considerada como una diáspora? A pesar que el concepto de diáspora es controversial en el campo de las ciencias sociales, me parece que es apropiado como metáfora que ayuda a comprender las prácticas y procesos transnacionales de comunicación entre las comunidades migrantes y sus comunidades de origen en El Salvador. La noción de diáspora implica, además, una consideración sobre las maneras en las que se transforman las identidades colectivas y nacionales, y se configuran nuevas identidades híbridas personales y colectivas. En esta discusión considero central la perspectiva de Clifford, para quien el término diáspora es un significante, no simplemente de transnacionalismo y movimiento, sino que al mismo tiempo expresa las luchas políticas por definir las características específicas de una comunidad en un contexto histórico de desplazamiento (Clifford, 1994: 308).

Sin duda, la experiencia de migración supone un desplazamiento físico y un proceso de desterritorialización o desanclaje cultural que se reterritorializa en un nuevo sistema social (Giddens, 1991:141). De esta forma, la organización de una comunidad en la diáspora genera nuevas condiciones en las cuales elementos de clase, raza, religión, sexo, etnicidad y pertenencia generacional se interrelacionan en la construcción de nuevas identidades híbridas (García Canclini, 2001:96). De igual forma, es importante analizar cómo los diferentes medios de comunicación colectiva: programas de televisión y radio diseñados para las comunidades en la diáspora, música popular, periódicos, revistas, videos caseros, teléfonos celulares, sitios web, entre otros, crean y mantienen recursos simbólicos para la producción y negociación de identidades colectivas en el espacio social transnacional (Karim, 2003:2).

\section{Materiales y métodos}

Este estudio se basa en una combinación de métodos de investigación. Por un lado, se ha utilizado el método de la etnografía de la comunicación, que consiste en una observación y descripción cercana de cómo los medios de comunicación son consumidos y la manera como éstos se insertan en las prácticas cotidianas de la gente. He tomado como referencia entrevistas y apuntes etnográficos realizados en el verano de 2004, específicamente en la comunidad salvadoreña inmigrante en el área metropolitana de Washington D.C., en los Estados Unidos. ${ }^{2}$

Por otro lado, desde las posibilidades de buscadores como yahoo y google he realizado una búsqueda de diferentes sitios y páginas web relacionadas con inmigrantes salvadoreños. Al mismo tiempo, me he auxiliado de sitios web que proveen diferentes enlaces y conexiones a otros sitios de la diáspora salvadoreña. Estos materiales recopilados han sido analizados desde la metodología del análisis textual, y tratando de establecer ciertas tendencias y características de contenidos y formatos de las auto-representaciones de grupos migrantes en el ciberespacio. 


\section{Resultados y discusión}

\section{Diáspora salvadoreña y usos del Internet}

Estudios sobre los usos del Internet por parte de comunidades migrantes se han enfocado en procesos de auto-representación de las voces y perspectivas de los migrantes, y en cómo las posibilidades de comunicación que provee el Internet se constituyen en un elemento central en la producción y mantenimiento de identidades colectivas en el ciberespacio (Mitra, 2001:29). Si bien no existe un consenso sobre los significados y el sentido del concepto de "comunidades virtuales", considero que es un término útil para evaluar y describir los procesos y prácticas de comunicación por medio del Internet y otras tecnologías de información y comunicación, especialmente los procesos que tienen lugar en el espacio social transnacional.

Tyner y Kuhlke (2000) proponen cuatro categorías para analizar los procesos de comunicación de las comunidades de la diáspora que se desarrollan por medio del Internet: intra-diáspora, inter-diáspora, diáspora y país de origen, diáspora y sociedad receptora. El nivel de intradiáspora incluye sitios web y usos del Internet entre los migrantes que residen en una comunidad específica o espacios cercanos de interacción social. Por ejemplo, una joven inmigrante salvadoreña que reside en el área metropolitana de Washington D.C. comenta: "yo uso mucho el Internet en el trabajo y también en el plano personal para comunicarme con mis amigos a través del Internet, además yo tengo todas mis cosas en el Internet”3. Así, para esta joven inmigrante, el Internet cumple una doble función: desempeño de su trabajo y como plataforma desde donde almacena sus informaciones, documentos, fotos, datos, etc., y mecanismo para comunicarse con sus redes sociales inmediatas.

Un ejemplo de sitio web de la diáspora salvadoreña en este nivel de intra-diáspora es Centro Deportivo (www.centrodeportivo.com). Este sitio fue desarrollado en 2001 por un joven salvadoreño que vive en el área de Washington D.C. La idea original era crear un sitio web dedicado exclusivamente a brindar información sobre fútbol. Sin embargo, el nivel de aceptación y popularidad del sitio web amplió su orientación a otras áreas como eventos sociales, culturales, religiosos, políticos y musicales de la comunidad salvadoreña y latina en esa área de Washington D.C. De hecho, a muchas personas les interesó este sitio web porque encontraban una gran cantidad de fotografías de los partidos de fútbol de sus comunidades, y de alguna manera se experimentaba un proceso de reconocimiento social en el espacio público del Internet. De esta forma, estos sitios en la web se constituyen en espacios de reconocimiento colectivo y también en espacios donde se mezclan algunas características de la cultura visual con las tradiciones de la cultura oral que prevalece en algunos inmigrantes salvadoreños. Al mismo tiempo, este sitio web puede incluirse en la segunda categoría de comunicación en el nivel de la inter-diáspora, pues de acuerdo al administrador de este sitio, recibe correos electrónicos de salvadoreños inmigrantes en diferentes ciudades de los Estados Unidos.

Otros sitios web de la diáspora salvadoreña podrían ser considerados dentro de la categoría de inter-diáspora, por ejemplo el sitio www.guanacos.com. Este sitio fue lanzado en 2004 y ha sido un instrumento muy útil de comunicación para salvadoreños dispersos alrededor del 
mundo. El sitio tiene información muy diversa: temas de migración, salud, turismo, cultura, deporte, música y venta de inmuebles. El sitio, además, tiene un enlace con el programa "la charamusca", www.lacharamusca.net, que se transmite semanalmente desde Los Estados Unidos. "La charamusca" es un programa en el que se habla sobre eventos culturales y tradiciones salvadoreñas como las fiestas patronales de diferentes pueblos, las memorias de la navidad y las historias personales de salvadoreños que viven en el exterior. El sitio web de "la charamusca" ofrece podcast de audio y video, un blog, recibe mensajes de texto y correos de voz, y contiene un mapa virtual de su audiencia alrededor del mundo.

Otro sitio web con características multimedia es www.salvadorenosenlinea.com, que presenta información sobre El Salvador, comidas típicas, leyendas y personajes de la tradición oral salvadoreña. Por otro lado, este sitio web cuenta con la capacidad de escuchar una radio local y ver en línea la señal de un canal local de la ciudad de Usulután, El Salvador. El sitio web http://cipotes.net ofrece una opción donde la diáspora salvadoreña tiene la oportunidad de participar y discutir sobre diferentes temas y noticias de actualidad de El Salvador. Este sitio abre diferentes chats y foros en línea para que los salvadoreños en la diáspora puedan expresar sus opiniones sobre temas sociales y políticos. Hasta enero de 2008, este sitio registraba un total de 734 miembros, en su mayoría salvadoreños migrantes que viven en diferentes países del mundo. Sin duda, este sitio web no sólo ofrece una opción para contactos individuales entre los salvadoreños en la diáspora, sino que además se convierte en un espacio particular en el que se despliegan y refuerzan características específicas de un sentido de identidad nacional salvadoreña.

Con el objetivo de establecer un medio de interconexión entre la diáspora salvadoreña se ha establecido el sitio www.salvadorenosenelmundo.org, administrado por la organización Salvadoreños en el Mundo con sede en la ciudad de Los Ángeles, Estados Unidos. Este sitio web contiene información sobre El Salvador, la diáspora salvadoreña, la cantidad de remesas que envían los salvadoreños en el exterior y noticias de actualidad. También cuenta con una serie de foros en línea para que los salvadoreños puedan expresar sus opiniones. En enero de 2008 este sitio contaba con foros sobre los siguientes temas: política salvadoreña, organizaciones salvadoreñas y alrededor del mundo, el voto de los salvadoreños en el exterior, arte y cultura, temas sobre la mujer y temáticas sobre migración. A pesar de la diversidad de temas que se proponen para comentarios y discusión, hasta ahora la participación en estos foros es muy baja. Por otra parte, la organización Salvadoreños en el Mundo ha sido la principal promotora de convenciones anuales de salvadoreños en la diáspora. Además, lidera la búsqueda de apoyos políticos y sociales para que se reconozca el derecho a participar en el proceso electoral a los salvadoreños en el exterior.

En el ámbito de los medios masivos de comunicación, en diferentes ciudades de los Estados Unidos se producen y transmiten diferentes programas de radio que están dirigidos hacia la comunidad salvadoreña inmigrante. Además, algunos de estos programas pueden ser escuchados por medio del Internet, de manera que pueden llegar a una audiencia más amplia y a la diáspora salvadoreña en diferentes países. Por ejemplo, en el área metropolitana de Washington D.C. existen dos estaciones de radio en español que tienen una importante audiencia en la comunidad salvadoreña: radio América (www.radioamerica.net) y radio La Campeona (www.lacampeona1420.com). Dada las posibilidades de escuchar estas 
emisoras en el Internet, éstas suelen recibir llamadas telefónicas y correos electrónicos desde otras ciudades en los Estados Unidos, incluso desde otros países. Una locutora de radio La Campeona comenta acerca de estas interacciones mediáticas que se dan al nivel de inter-diáspora: "hemos recibido llamadas telefónicas de gente fuera del estado, de North Carolina, California, Houston. Además nuestros oyentes nos recomiendan a otras personas y a la gente le gusta mucho que por medio del Internet no sólo nos pueden escuchar, sino también ver a través de la webcam que tenemos en la radio"“.

Otro sitio web que posibilita la comunicación entre la diáspora salvadoreña fue lanzado en 2006 por la Casa de la Cultura de El Salvador en Los Ángeles. Esta organización promovió la creación de la radio Pipiles (www.radiopipiles.org), que se define como una radio que transmite exclusivamente en Internet y busca convertirse en un punto de enlace para la diáspora salvadoreña alrededor del mundo. Esta radio está basada en Los Ángeles, California y espera poder ser un medio de intercomunicación entre los salvadoreños del sur de California, de otras ciudades del país y de otros países. Además, este proyecto radiofónico cuenta con el apoyo de organizaciones sociales locales y voluntarios comunitarios de Los Ángeles.

Desde la perspectiva de procesos de comunicación entre la diáspora y el país de origen existen diferentes sitios web y medios de comunicación masivos a los que se puede tener acceso desde el Internet. Algunos inmigrantes salvadoreños afirman que la posibilidad de leer o escuchar algunos medios de comunicación de El Salvador les permite sentir un lazo de contacto y comunicación con lo que sucede en su país. Hasta hace algunos años, solamente algunos periódicos -como El Diario de Hoy (www.elsalvador.com) y La Prensa Gráfica (www. laprensagrafica.com) - estaban también disponibles en el Internet; sin embargo, hoy en día hay más opciones de medios impresos salvadoreños que tienen una versión en la web. De hecho, estos dos periódicos salvadoreños han incorporado desde 2007 un nuevo elemento en sus sitios web: el e-paper, que consiste en un formato electrónico que permite leer el periódico tal y como aparece en la versión impresa completa. De acuerdo a algunos informes, esta decisión se produjo por la misma presión de la diáspora salvadoreña que quiere tener acceso a la misma versión impresa que circula en El Salvador. Esto revela la importante influencia de la diáspora salvadoreña en el consumo de periódicos por medio del Internet. De acuerdo a algunas estimaciones, entre el 70 y $80 \%$ de las visitas a los sitios web de estos dos periódicos provienen de personas que se encuentran fuera del país.

Adicionalmente, muchos salvadoreños inmigrantes demandan de los medios de comunicación de El Salvador más noticias locales, particularmente de sus diferentes pueblos y departamentos de origen. Además de los periódicos, la diáspora salvadoreña puede acceder a diferentes estaciones de radio y de televisión de El Salvador por medio del Internet. Por lo tanto, las opciones de estar en contacto con el país y las comunidades de origen a través de los medios de comunicación con presencia en el Internet se han ampliado significativamente en los últimos años.

Desde la perspectiva de las identidades locales, las posibilidades de comunicación del Internet permiten establecer y potenciar nuevos vínculos entre comunidades inmigrantes y sus familias, amigos y comunidades de origen. Un caso que ilustra esta dinámica es el sitio 
www.intipucacity.com, el cual busca interconectar la ciudad de Intipucá en el departamento de La Unión en El Salvador con los inmigrantes que viven en el área de Washington D.C. Este sitio web contiene noticias, fotografías de eventos sociales y culturales, comentarios, espacios de chat y otras actividades que ocurren en ambos espacios sociales: en Intipucá y Washington D.C. Este sitio web pudiera ser considerado como una "comunidad virtual" o "ciudad virtual" que permite la interacción transnacional de dos comunidades y promueve elementos que son considerados propios de la identidad colectiva de Intipucá. El fundador y administrador de este sitio web que vive en Estados Unidos comenta: "yo tengo algunos patrocinadores y una persona que me envía información desde Intipucá. La gente usa mucho el chat, sobre todo desde allá para acá. El chat lo usan sobre todo los jóvenes”.

En el mismo nivel de relación diáspora y país de origen, diferentes asociaciones $u$ organizaciones de salvadoreños en la diáspora han creado sus propias páginas web para fomentar la participación de la comunidad inmigrante y mantener lazos de contacto con el país de origen. De las diferentes organizaciones de salvadoreños existentes, algunos sitios web que he podido encontrar y analizar son los siguientes. El sitio de la diáspora salvadoreña en Canadá (www.elsalvadorencanada.com), que fue lanzado en 2006 y pretende ser un portal de comunicación e interacción para los salvadoreños que viven en Canadá. Hasta enero de 2008, este sitio registraba en sus estadísticas un total de 231 miembros inscritos. El sitio permite subir videos, bajar música y participar en espacios de chat y foros de discusión. Otra comunidad migrante que cuenta con una presencia en el Internet son los salvadoreños residentes en Suecia (www.conase.se). Este sitio provee información y noticias de El Salvador y América Latina, eventos locales en Suecia, fotografías y datos generales sobre ambos países. De igual manera existe una página web, aunque con poco desarrollo en los contenidos y herramientas, de los salvadoreños residentes en Italia (http://web.tiscali. it/comunidad/gruvisal.htm).

En los Estados Unidos también se pueden encontrar diferentes sitios web que se usan como mediofundamental parainformar sobreactividades, y presentar opiniones y representaciones de parte de organizaciones de inmigrantes salvadoreños. Entre estos sitios está el de la Asociación de Salvadoreños en Los Angeles (ASOSAL), www.asosal.org. También está el sitio de la Asociación Internacional de Mujeres Salvadoreñas, www.aimsal.org, asociación que fue fundada en 1996 y busca ayudar en proyectos de desarrollo -particularmente a través de becas educativas- a las comunidades de origen de sus integrantes. Por otro lado, algunos grupos de inmigrantes salvadoreños están usando cada vez más los blogs como plataforma para compartir puntos de vista y generar discusión en este nivel de esfera pública. Algunos ejemplos de estos blogs son: http://elsalvadorcomite.blogspot.com. Este blog incluye comentarios, noticias y artículos con posiciones muy críticas frente al gobierno del presidente Antonio Saca. Otro blog que está orientado a la diáspora salvadoreña es el de la Asociación de Salvadoreños en el Mundo, http://salvadorenosenelmundo.blogspot.com. Este blog ofrece oportunidades para enviar comentarios y participar en diferentes debates sobre temas de actualidad y de interés para los inmigrantes salvadoreños de la diáspora.

Finalmente, en el nivel de análisis de la interacción diáspora y sociedad receptora de los inmigrantes, hay muy pocos ejemplos en el caso de los salvadoreños en el exterior. En cierta 
forma, una de las dificultades es que los sitios o páginas web que se han creado están en español y buscan promover sobre todo el nivel de interacción intra-diáspora, inter-diáspora y diáspora con El Salvador. No obstante, he encontrado algunos sitios que podrían ser considerados dentro de esta categoría de interacción con la sociedad receptora. Algunos inmigrantes, especialmente jóvenes o hijos de inmigrantes de la primera generación, están empleando cada vez más los nuevos mecanismos de interacción y creación de redes sociales que ofrece el Internet como blogs, Facebook, Myspace, entre otras opciones. Por ejemplo, un grupo de jóvenes salvadoreños que viven en los Estados Unidos, han creado un sitio en Myspace desde el cual intentan llegar a un público bilingüe o que usa más frecuentemente el inglés: www.myspace.com/salvadorenosenelmundo. Este sitio ofrece contenidos en el idioma inglés, información sobre eventos comunitarios de comunidades inmigrantes de salvadoreños, galería de fotografías y enlaces a videos sobre El Salvador que están disponibles en YouTube. Otros enlaces de este sitio refieren a un sitio de Homies Unidos en Los Ángeles que intenta promover opciones y alternativas creativas para evitar la violencia, las pandillas juveniles, y el consumo de drogas entre los jóvenes latinos. Otro sitio es http://groups.myspace.com/salvadorpride, que integra a 6,094 miembros registrados de acuerdo a las estadísticas del sitio hasta el mes de enero de 2008. Este grupo intercambia fotos, facilita la creación de redes de amigos y amplía otras redes sociales especialmente entre los jóvenes. Este tipo de redes sociales integra jóvenes de origen salvadoreño, pero también otras personas que pueden compartir intereses sociales y culturales. Este nivel de interacción, en el que se usan formas virtuales de comunicación, representa también una forma de incidir en la configuración de identidades individuales y colectivas entre personas y grupos migrantes en el ciberespacio.

\section{Reflexiones finales}

Las categorías de análisis sobre las formas de interacción que establecen las comunidades en la diáspora a través del Internet son útiles para distinguir algunos espacios y actores en dichos procesos. Sin embargo, es importante señalar que algunos de estos niveles de interacción se traslapan y adquieren su propio dinamismo en las prácticas de uso y de apropiación de los migrantes. Hace falta todavía profundizar en el análisis sobre los usos y sentidos que los migrantes le dan a sus prácticas de comunicación a través del Internet. Algunos métodos, como la etnografía del Internet, o la observación participante de sitios web como algunos de Myspace y Facebook-orientados hacia personas o comunidades migrantesnos pueden dar mayores elementos de reflexión en el campo de la comunicación.

El estudio de los procesos de comunicación en la experiencia de las comunidades migrantes aporta nuevos elementos para el análisis de la integración social en nuestras sociedades. Al mismo tiempo, plantea nuevos desafíos desde la configuración de complejas sociedades transnacionales (Giddens, 1984:139). Es evidente que los medios de comunicación masivos han sido fundamentales para generar nuevas experiencias de convivencia y prácticas en las comunidades migrantes, ya sean en el nivel de co-presencia, interacción mediada o quasi-mediada (Thompson, 1995:84). Los medios tradicionales: radio, televisión, periódicos y revistas, han sido los mecanismos de contacto de muchas comunidades de salvadoreños migrantes con sus países de origen, especialmente cuando estos medios han comenzado a 
estar accesibles en el Internet. Más recientemente, la creación de sitios y páginas en la web han ofrecido nuevos mecanismos para expresar y recibir nuevas perspectivas ideológicas, opiniones y experiencias. Por esta razón, me parece importante continuar estudios que puedan ir dibujando un mapa de la presencia de los grupos migrantes en el ciberespacio. Al mismo tiempo, han surgido nuevas posibilidades de medios de comunicación que utilizan únicamente el Internet, tal es el caso de la Radio Pipil, que intenta interrelacionar a la diáspora salvadoreña. Todavía con mayores posibilidades de acceso y uso son los blogs y plataformas de redes sociales como Myspace y Facebook, las que ofrecen posibilidades para la creación de nuevas expresiones y representaciones de las comunidades migrantes. Aunque por ahora pareciera que el uso de estas plataformas es muy limitado entre la comunidad salvadoreña inmigrante, representa un potencial de interacción y promoción de elementos identitarios para personas y grupos migrantes.

Obviamente, no podemos plantear estas posibilidades de usos del Internet entre las comunidades migrantes sin hacer algunas reflexiones del problema de la brecha digital. ${ }^{6}$ Aunque algunas personas piensan que no tiene sentido hablar de la brecha digital, este fenómeno debe analizarse desde su complejidad y sus consecuencias. Tal como lo plantea Castells, el problema de la brecha digital no es únicamente una cuestión de conexión y usos del Internet, sino sobre todo, de entender de qué maneras la brecha digital genera una distribución desigual de conocimiento, poder y capacidad de vinculaciones sociales que dependen cada vez más de las nuevas tecnologías de información y comunicación. (Castells, 2001:265). De esta forma, es necesario analizar cómo los migrantes en las sociedades receptoras tienen o no acceso al Internet y relacionar esa situación con el acceso tan limitado que tienen países emisores de migrantes como El Salvador. ${ }^{7}$ De acuerdo a las estadísticas proporcionadas por la Unión Internacional de Telecomunicaciones (ITU), en 2006 El Salvador tenía 127,410 suscriptores a Internet (1.85 por cada 100 habitantes) y 637,000 usuarios de Internet (9.26 por cada 100 habitantes). Por otro lado, Escobar (2000:57) subraya que la comprensión de la brecha digital demanda la incorporación de una agenda de investigación sobre la cibercultura. Esta agenda tendría que incluir la indagación sobre los nuevos discursos, metodologías, prácticas y economía política de las tendencias originadas desde la cibercultura.

En resumen, las diversas formas de comunicación que tienen como base el Internet: medios de comunicación, sitios web, páginas web de organizaciones, blogs, espacios de chat, intercambio de materiales y otras plataformas virtuales para redes sociales, ofrecen nuevas posibilidades para la expresión de representaciones desde la perspectiva de los inmigrantes. Aunque algunas de estas posibilidades todavía tienen un uso limitado entre las comunidades migrantes, éstas pueden constituirse en espacios claves de expresión de identidades. Al mismo tiempo, las narrativas, historias, opiniones y preocupaciones de los migrantes que aparecen en estos sitios virtuales se entrelazan con una serie de símbolos de identidades colectivas y demandas de reconocimiento social y político. En el caso de El Salvador, hay todavía una deuda, de parte de la sociedad y del sistema político salvadoreño, de reconocer los derechos ciudadanos y de participación política de los migrantes que contribuyen significativamente a la economía del país por medio de las remesas familiares. Hasta el año 2007, las remesas familiares recibidas en El Salvador han alcanzado el monto de US\$3,695 millones de acuerdo a las estadísticas oficiales del Banco Central de Reserva de El Salvador. ${ }^{8}$ 
De cara al futuro sería importante comparar las prácticas de comunicación basadas en el Internet que realizan los inmigrantes salvadoreños con las realizadas por migrantes de otras nacionalidades, particularmente de otros países centroamericanos. Por otra parte, hace falta profundizar en el análisis denso de las expresiones de identidad que se construyen, se transforman y se confrontan en luchas simbólicas que toman lugar en el ciberespacio. También hace falta explorar cómo los discursos y las narrativas oficiales entran en disputa con nuevas visiones, experiencias y necesidades de las comunidades migrantes que se sienten a veces sin referentes, sin raíces y sin el respaldo de un estado-nación. O por el contrario, habría que valorar hasta qué punto nuevas plataformas de redes sociales en el Internet generan más dispersión de las comunidades migrantes, y en definitiva, promueven una reducción sensible o incluso invisibilidad de los migrantes en las esferas mediadas de discusión pública. En última instancia, hace falta seguir dibujando los nuevos mapas culturales y las nuevas identidades colectivas que los grupos migrantes trazan desde sus prácticas de comunicación en el ciberespacio.

\section{Notas}

1 Ministerio de Relaciones Exteriores de El Salvador, disponible en: http://www.rree.gob.sv/sitio/sitiowebrree.nsf/pages/ ssalvext asuntoscomunitarios. Consultado el 14 de octubre de 2007.

2 Ver Benítez, J.L. (2005). Disertación doctoral: Communication and collective identities in the transnational social space: a media ethnography of the Salvadoran immigrant community in the Washington D.C. metropolitan area. (Ohio University). Disponible en el sitio: http://www.ohiolink.edu/etd/view.cgi?acc num=ohiou1121349361

3 Martínez, A. (2004). Comunicación personal.

4 Parada, V. (2004). Comunicación personal.

5 Velásquez, C. (2004). Comunicación personal.

6 Ver Benitez, J.L. (2006) Transnational dimension of the digital divide among Salvadoran immigrants in the Washington D.C. area. Global Networks 6 (2), 181-199.

7 Ver Pew Internet \& American Life Project, 2007. Disponible en: http://www.pewinternet.org/pdfs/Latinos Online March 14 2007.pdf.

8 Banco Central de Reserva de El Salvador. Comunicado No. 1/2008. Disponible en: http://www.bcr.gob.sv/publicaciones/ comunicados/2008/main comunicado12008.html

\section{Referencias bibliográficas}

BENITEZ, J.L. (2005). Communication and collective identities in the transnational social space: a media ethnography of the Salvadoran immigrant community in the Washington D.C. metropolitan area. Tesis doctoral. Athens, Ohio. Ohio University.

CASTELLS, M. (2001). The Internet galaxy: reflections on the Internet, business and society. Oxford: Oxford University Press.

CLIFFORD, J. (1994). “Diaspora”. En Cultural Anthropology 9 No. 3, 302-338.

ESCOBAR, A. (2000). "Welcome to Cyberia: notes on the anthropology of cyberculture". En The cyberculture reader, Bell, D \& Kennedy, B (eds). London: Routledge.

GARCÍA CANCLINI, N. (2001). Consumer and citizens. Globalization and multicultural conflicts. Minneapolis: University of Minnesota Press.

GIDDENS, A. (1984). The constitution of society. Berkley: University of California Press.

GIDDENS, A. (1991). Modernity and self-identity. Stanford: Stanford University Press.

KARIM, K. (2003). "Mapping diasporic mediascape” En The media of diaspora, Karim, K. 
(ed). London: Routledge.

KOSER, K. (2007). International migration. A very short introduction. Oxford: Oxford University Press.

MITRA, A. (2001). "Marginal voices in cyberspace”. En New Media \& Society 3, No. 1, 2948.

THOMPSON, J.B. (1995). The media and modernity. A social theory of the media. Cambridge: Cambridge University Press.

TYNER, J.A. y KUHLKE, O. (2000). "Pan-national identities: representations of the Philippine diaspora on the World Wide Web”. En Asian Pacific Viewpoint, Vol. 41, No. 3: 231-252. 